\title{
A control chart design used in supply chain management system
}

\author{
Jing Sun*, Masayuki Matsui \\ The University of Electro-Communications, 1-5-1 Chofugaoka, Chofu-shi, Tokyo, 182-8585, Japan
}

(Received December 13 2007, Accepted March 10 2008)

\begin{abstract}
This paper presents a design of the $\bar{x}$ control chart used in supply chain management system to improve customer satisfaction of supplier. Recently, due to ever increasing competition in supplier markets, it is demanded to offer the low-cost and high quality product in short due time. Therefore, studying the balance of quality and the due time and cost has become a new problem to supplier. In short run process, the time of treating an assignable cause (ACT time) is an important effect to the total cost and due time. To resolve the above problem, in this research, first a design of the control chart is developed in which the ACT time was considered. Then, from an economic viewpoint, the mathematical formulations which correspond to the design are shown. Finally, by numerically consideration, the balance of quality (control limits width) and due time and the total expectation cost is discussed, and the important relations between the due time, ACT time and the total expectation cost are investigated.
\end{abstract}

Keywords: supply chain management, PDCA design, short due time, economic evaluation, control chart model

\section{Introduction}

In today's supplier chain management system shown in Fig. 1, because of increasing competition in supplier markets, the supplier (or Manufacturer) is demand to offer low cost and high quality products to maker in short due time.

In this background, it have become more important to study the balance of quality and the due time and cost. Therefore, in this research, we present a design of the $\bar{x}$ control chart in view of quality, due time and cost for supplier to improve customer satisfaction.

Sun, Tsubaki and Matsui ${ }^{[15]}$ have defined and considered the PDCA model with tardiness penalty of the $\bar{x}$ control chart based on the case which starts from deciding the control lines.

These days, business environments have become competitive, and the due time of products has shortened. In short run process, the time of treating the assignable cause (ACT time) is an important effect to the total cost and due time. However, the ACT time was not considered in Sun, Tsubaki and Matsui ${ }^{[15]}$.

In this research, first the PDCA design of the $\bar{x}$ control chart is developed in which the ACT time was considered. Then, from an economic viewpoint, the mathematical formulations which correspond to the design are shown. Finally, by numerically consideration, the balance of quality (control limits width), due time and the total expectation cost is discussed, and the important relations between the due time, ACT time and the total expectation cost are investigated.

\footnotetext{
* Corresponding author. Tel: +81-424-43 5261; fax: +81-424-43 5821.

E-mail address: son@se.uec.ac.jp.
} 


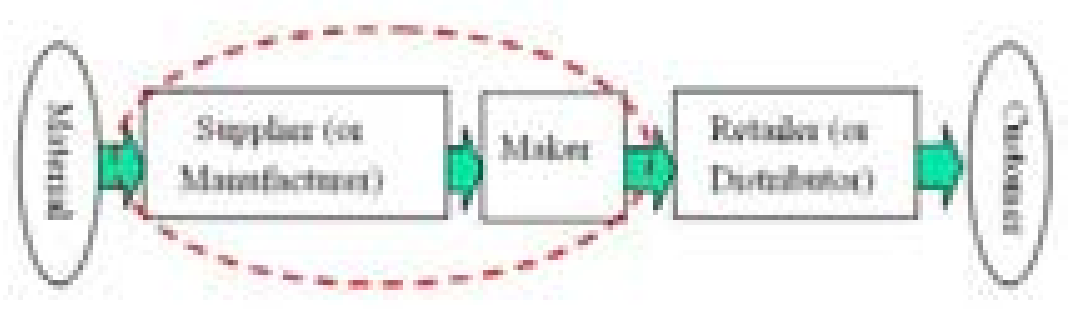

Fig. 1. The supply chain management system

\section{Review of the PDCA design of control charts}

Recently, to improve supplier quality, there has been an increased interest in IT (information technology) control charts which are used to monitor online production processes.

On the other hand, the concept of the PDCA (Plan, Do, Check and Act) cycle originally developed by Walter Shewhart during the 1930 's ${ }^{[12]}$, and implemented and promoted very effectively from the 1950 s by W. Edwards Deming, has consequently played a key role in the field of quality control. After that, 'the Deming Cycle' is developed not only as a quality cycle in the quality control field, but also as a management cycle which has played a key role in all of the management fields ${ }^{[8]}$.

Recently, to clarify the certain points and improve quality more efficiently and effectively, detailed attention has been paid to the PDCA procedure which starts with the check stage for the quality management activity to allow more efficient use of energy in the industry $([6,10,13]$, etc.).

The classical definitions of the control chart's CAPD procedures are known. Recently, the evaluation of the economy of this control chart's PDCA procedures is connected with "daily management".

Since Duncan's pioneering work ${ }^{[4]}$, many studies have been developed to serve different purposes for the economic model of control charts. From the viewpoint of the production run, Gibra ${ }^{[5]}$, Ladany and Bedi ${ }^{[1]}$, Jones and Chase ${ }^{[7]}$, Saniga ${ }^{[11]}$ have considered the economic statistical model of the $\bar{x}$ control chart for the infinite-length horizon; Crowder ${ }^{[3]}$, Del Castillo and Montgomery ${ }^{[2]}$ have considered models of the control chart for short run cases.

However, the model from the viewpoint of the PDCA process was not considered.

Therefore, Sun, Tsubaki and Matsui ${ }^{[14]}$ defined the PDCA model of the $\bar{x}$ control chart; and then Sun, Tsubaki and Matsui ${ }^{[15]}$ considered the PDCA model of the $\bar{x}$ control chart with tardiness penalty.

In the short run production process, the ACT time is an important effect to the cost and the due time. Therefore, the aim of this paper is to present and study the PDCA design in which the ACT time was considered.

\section{The design}

To improve customer satisfaction of supplier in SCM, this paper presents a PDCA design of the control chart in view of quality, due time and cost. The PDCA design is set up based on the case which starts from deciding the control lines of the $\bar{x}$ chart, in which the ACT time was considered. The procedure (Plan, Do, Check and Act) of the design is defined as follows:

Definition 1. Assuming the production process is monitored by the $\bar{x}$ control chart from this point forward. PLAN is defined as constructing the control lines (center line and upper and lower control limit) for future management. DO is defined as sampling and plotting on the $\bar{x}$ control chart for monitoring the process quality with the decided control lines in the PLAN procedure.

Definition 2. CHECK is defined as judging whether the process is in an in-control state by the result of the comparison between the point plotted on the $\bar{x}$ chart and the control limits (upper and lower control limit). Finally, ACT is defined as correcting the assignable cause for restoring the in-control state. 


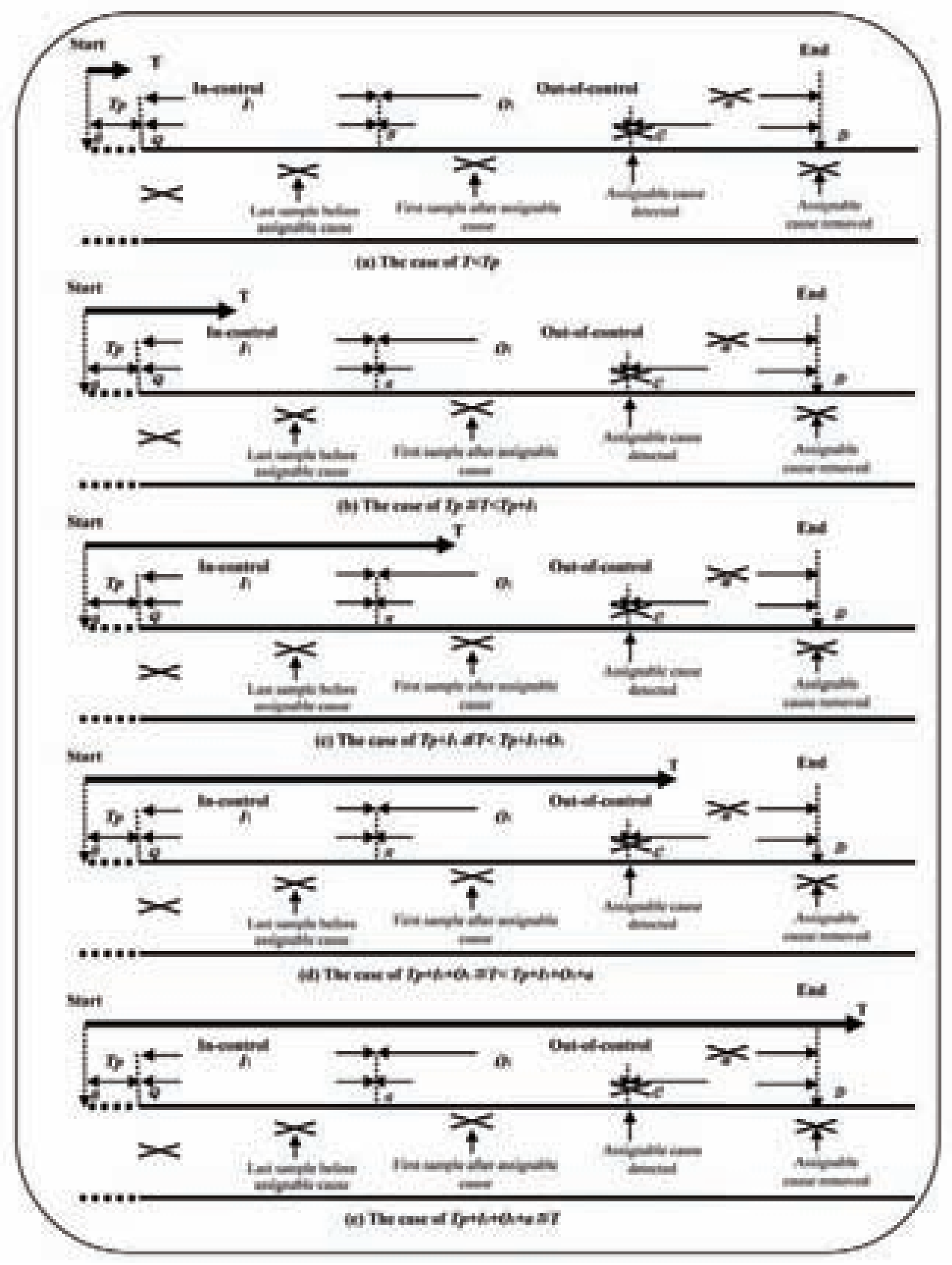

Fig. 2. Some of the time variables used in the CAPD model considered the ACT time 


\subsection{The assumption and the notation}

The assumptions of the design in this research are as follows:

(1) The production run length $T$ is short, and the process is repetitive.

(2) The random variables of in-control interval and out-of-control interval are assumed to be exponentially distributed with the mean $\lambda_{1}^{-1}$ and $\mu_{1}^{-1}$.

(3) The quality shift occurs in the middle of an interval between samples ${ }^{[9]}$

The notation used is as follows:

$C_{p}$ : expected cost of PLAN per unit time

$C_{d}$ : expected cost of DO per unit time

$C_{c}$ : expected cost of CHECK per unit time

$C_{a}$ : expected cost of ACT per unit time

$C_{t}$ : expected total cost per unit time

$n_{1}$ : the sample size per each sampling

$v_{1}$ : the sampling interval

$T$ : production run length

$T_{p}$ : the interval of PLAN

$I_{1}$ : period from $Q$ to $S$

$O_{1}$ : period from $S$ to $C$

$c_{0}$ : fixed sampling cost

$c_{1}$ : variable sampling cost

$c_{2}$ : cost of per unit time for checking the point plotted

$c_{3}$ : cost of a false alarm

$c_{4}$ : cost of restoring an in-control state

$c_{\beta p}$ : cost of per unit time for penalties delay of PLAN

$c_{\beta d}$ : cost of per unit time for penalties delay of DO

$c_{\beta c}$ : cost of per unit time for penalties delay of CHECK

$c_{\beta 1}$ : cost of per unit time for penalties delay of ACT

$f$ : number of samples taken during $T-T_{p}$

$\delta$ : size of the quality shift in the mean

$a$ : the ACT time

$\lambda_{1}^{-1}$ : mean of the $I_{1}$ period in the PDCA design

$\mu_{1}^{-1}$ : mean of the $O_{1}$ period in the PDCA design

$k$ : control limits width

$\phi_{1}$ : the value of $\operatorname{Pr} 0 \leq T<v_{1}$

$\phi_{2}:$ the value of $\operatorname{Pr} T_{p} \leq T<T_{p}+v_{1}$

\subsection{The mathematical formulations}

The evaluation function of this PDCA design is the expected total cost per unit time as follows:

$$
\begin{aligned}
C_{t(C A P D)} & =\frac{E[\text { cost percycle }]}{E[\text { cycle }(P D C A)]}=\frac{E[\text { cost percycle }]}{E\left[\min \left(T_{p}+I_{1}+O_{1}+a, T\right)\right]} \\
& =C_{p}+C_{d}+C_{c}+C_{a}
\end{aligned}
$$

It includes the cost of PLAN, DO, CHECK, ACT. Fig. 1 shows some of the time variables used in the PDCA design considered ACT time.

At the start of the PDCA model, a sufficient plan of deciding control lines is made in $T_{p}$ time. Therefore, it is thought that the PDCA model starts from the in-control state, because the process is managed by these control lines. Let the process start at the point of $Q$, and $S$ be the point in time at which the quality characteristic shifts to an out-of-control state as shown in Fig. 1. At the point of $C$ an assignable cause is detected for the first time by the $\bar{x}$ control chart. After that the assignable cause is corrected at the point of $D$. Here, 
the random variables $I_{1}$ and $O_{1}$ represent the interval from $Q$ to $S$ and the interval from $S$ to $C$. If $T_{p}$ denotes the time of PLAN and a denotes the time of ACT, then the time from the start of the production process until removing the assignable cause is equal to $T_{p}+I_{1}+O_{1}+a$.

The mathematical formulations correspond to this design are shown as follows:

$$
\begin{aligned}
C_{p} & =C_{p(s)}+C_{p(p)} \\
& =\left\{\left[\left(c_{0}+c_{1} n_{1}\right) / v_{1}\right] E\left[T_{p s 1}\right]+c_{\beta p \phi_{1}}\right\} / E[\text { cycle }] \\
C_{d} & =C_{d(s)}+C d(p) \\
& =\left\{\left[\left(c_{0}+c_{1} n_{1}\right) / v_{1}\right] E\left[T_{d s 1}\right]+c_{\beta d \phi_{2}}\right\} / E[\text { cycle }] \\
C_{c} & =C_{c(c)}+C_{c(e)}+C_{c(p)} \\
& =\left\{\left(c_{2} / v_{1}\right)\left(E[c y c l e]-E\left[T_{p s 1}\right]\right)+\left(c_{3} / v_{1}\right) \alpha E\left[\min \left(I_{1}, T-T_{p}\right)\right]\right. \\
& \left.+c_{\beta c} E\left[\left(T-T_{p}-I_{1}\right)^{+}-\left(T-T_{p}-I_{1}-O_{1}\right)^{+}\right]\right\} / E[c y c l e] \\
& \\
C_{a} & =C_{a(a)}+C_{a(p)} \\
& =\left\{c_{4} E\left[\left(T-T_{p}-I_{1}-O_{1}\right)^{+}-\left(T-T_{p}-I_{1}-O_{1}-a\right)^{+}\right]\right. \\
& \left.+c_{\beta a} \operatorname{Pr}\left\{T_{p}+I_{1}+O_{1} \leq T<T_{p}+I_{1}+O_{1}+a\right\}\right\} / E[\text { cycle }]
\end{aligned}
$$

The elements of costs of CHECK, ACT, PLAN and DO are shown as follows:

Table 1. The elements of costs of PLAN, DO, CHECK and ACT

\begin{tabular}{cll}
\hline Procedure & & \multicolumn{1}{c}{ Element } \\
\hline \multirow{2}{*}{ PLAN $\left(C_{p}\right)$} & $C_{p(s)}$ & cost of PLAN \\
& $C_{a(p)}$ & cost of the penalty for delaying the PLAN \\
\hline \multirow{2}{*}{ DO $\left(C_{d}\right)$} & $C_{d(s)}$ & cost of DO \\
& $C_{d(p)}$ & cost of the penalty for delaying the DO \\
\multirow{2}{*}{ CHECK $\left(C_{c}\right)$} & $C_{c(c)}$ & cost of CHECK \\
& $C_{c(e)}$ & cost of type I error \\
& $C_{c(p)}$ & cost of the penalty for delaying the CHECK \\
\hline \multirow{2}{*}{$\mathrm{ACT}\left(C_{a}\right)$} & $C_{a(a)}$ & cost of ACT \\
& $C_{a(p)}$ & cost of the penalty for delaying the ACT \\
\hline
\end{tabular}

If it is assumed that both the random variable $I_{1}, O_{1}$ are independent and exponentially distributed with mean $\lambda_{1}^{-1}, \mu_{1}^{-1}$, then combining equations (1), (2), (3), (4), (5), the expected total cost per unit time of the PDCA design considered the ACT time is shown as follows: 


$$
\begin{aligned}
C t= & \left\{\left(c_{0}+c_{1} n_{1}\right) / v_{1}\right)\left[T \phi_{1}+T_{p}\left(1-\phi_{1}\right)\right]+c_{\beta p} \phi_{1}+\left[\left(c_{0}+c_{1} n_{1}\right) / v_{1}\right] \\
& {\left[\frac{1}{\lambda_{1}-\mu_{1}}\left\{\frac{\mu_{1}}{\lambda_{1}}\left(e^{-\lambda_{1}\left(T-T_{p}-a\right)}-1\right)-\frac{\lambda_{1}}{\mu_{1}}\left(e^{-\mu_{1}\left(T-T_{p}-a\right)}-1\right)\right\}+T_{p}+a-\right.} \\
& \left.\left(T \phi_{1}+T_{p}\left(1-\phi_{1}\right)\right)\right]+c_{\beta d} \phi_{2}+\left(c_{2} / v_{1}\right)\left[\frac { 1 } { \lambda _ { 1 } - \mu _ { 1 } } \left\{\frac{\mu_{1}}{\lambda_{1}}\left(e^{-\lambda_{1}\left(T-T_{p}-a\right)}-1\right)-\right.\right. \\
& \left.\left.\frac{\lambda_{1}}{\mu_{1}}\left(e^{-\mu_{1}\left(T-T_{p}-a\right)}-1\right)\right\}+T_{p}+a-\left(T \phi_{1}+T_{p}\left(1-\phi_{1}\right)\right)\right]+ \\
& \left(c_{3} / v_{1}\right) \alpha \frac{1}{\lambda_{1}}\left(1-e^{-\lambda_{1}\left(T-T_{p}\right)}\right)+c_{\beta c}\left[\frac{1}{\mu_{1}}+\frac{1}{\lambda_{1}-\mu_{1}}\left(e^{-\lambda_{1}\left(T-T_{p}\right)}-\right.\right. \\
& \left.\left.\frac{\lambda_{1}}{\mu_{1}} e^{-\mu_{1}\left(T-T_{p}\right)}\right)\right]+c_{4}\left[a+\frac{1}{\lambda_{1}-\mu_{1}}\left(\frac{\lambda_{1}}{\mu_{1}} e^{-\mu_{1}\left(T-T_{p}\right)}\left(1-e^{\mu_{1} a}\right)-\right.\right. \\
& \left.\left.\frac{\mu_{1}}{\lambda_{1}} e^{-\lambda_{1}\left(T-T_{p}\right)}\left(1-e^{\lambda_{1} a}\right)\right)\right]+c_{\beta a}\left[\frac { \lambda _ { 1 } \mu _ { 1 } } { \lambda _ { 1 } - \mu _ { 1 } } \left(-\frac{1}{\mu_{1}} e^{-\mu_{1}\left(T-T_{p}\right)}\left(1-e^{\mu_{1} a}\right)+\right.\right. \\
& \left.\left.\left.\frac{1}{\lambda_{1}} e^{-\lambda_{1}\left(T-T_{p}\right)}\left(1-e^{\lambda_{1} a}\right)\right)\right]\right\} /\left[\frac { 1 } { \lambda _ { 1 } - \mu _ { 1 } } \left\{\frac{\mu_{1}}{\lambda_{1}}\left(e^{\lambda_{1}\left(T-T_{p}-a\right)}-1\right)-\right.\right. \\
& \left.\left.\frac{\lambda_{1}}{\mu_{1}}\left(e^{-\mu_{1}\left(T-T_{p}-a\right)}-1\right)\right\}+T_{p}+a\right]
\end{aligned}
$$

In this paper, the statistical hypothesis is that the mean equals a standard value. When $\Phi(Z)=$ $e^{-Z^{2} / 2} / \sqrt{2 \pi}$ is the standard normal density, $\alpha$ (the type I error probability) and $P_{o}$ (power) of the $\bar{x}$ control chart are given by ${ }^{[2]}$,

$$
\begin{aligned}
& \alpha=2 \int_{k_{i}}^{\infty} \Phi(Z) d Z \\
& P_{o}=\int_{-\infty}^{-k-\delta_{i} \sqrt{n_{i}}} \Phi(Z) d Z+\int_{-k-\delta_{i} \sqrt{n_{i}}}^{\infty} \Phi(Z) d Z .
\end{aligned}
$$

Where $\delta$ is the size of the quality shift in the mean, and $k$ is control limits width. We assume that the outof-control period $O_{1}$ is an exponential random variable with the mean $\mu_{1}^{-1}$. In this paper we use Ladany and Bedi's assumption (1976) that the shift occurs in the middle of an interval between samples and set $\mu_{1}^{-1}$ as follows:

$$
\mu_{1}^{-1}=v_{1}\left(1 / P_{0}-1\right)+v_{2} / 2=v_{1}\left(1 / P_{0}-1 / 2\right)
$$

\section{Numerical experiments}

In this section, for improving customer satisfaction of supplier, first we study the relations between the due time and ACT time and the total expectation cost, then we investigate the balance of control limits width $(k)$ and due time $(T)$ and the total expectation cost $(C t)$ by numerically analyzing the above design. Where, $c_{0}=1, c_{1}=0.1, c_{2}=10, c_{3}=50, c_{4}=25, c_{\beta a}=c_{\beta p}=c_{\beta d}=200, c_{\beta c}=2400, n_{1}=4, v_{1}=0.0316$, $T_{p}=1, \phi_{1}=0.01, \phi_{2}=0.001, \lambda_{1}=1$. The parameters are based on a real situation. We evaluate a wide range of possible values. Because the result is approximated, we show the results of the above case as an example.

\subsection{The balance of $k, T$ and $C t$}

Fig. 3 shows the balance of the quality (control limits width) and due time and the total expectation cost of the above case, which is useful for setting the optimal due time and control limits width to the supplier.

From Fig. 3, it can be understood that this tables are divided into two areas by the changed control limits width: in the colorlessness area, the expected total cost per unit time $(C t)$ increases with the increase of due 
time $(T)$; in the blue area, the expected total cost per unit time $(C t)$ decreases with the increase of due time $(T)$.

From Fig. 3 and Fig. 4, it can be noted that the expected total cost per unit time $(C t)$ increases with the increase of control limits width $(k)$. This is because that the cost of defective goods increases by the increase of control limits width.

From Fig. 4, it also can be understand that a longer due time should be set when the high quality is demanded, while a shorter due time should be set when the low quality is demanded from an economic aspect.

In addition, to clarify it more, we also show the Fig. 5 which is the same as the case of Fig. 3.

\begin{tabular}{|c|c|c|c|c|c|c|c|c|c|c|c|c|c|c|}
\hline \multirow{2}{*}{7} & \\
\hline & 171 & $x$ & $\mathbb{B}$ & 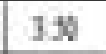 & 1 & $y$ & $4 y$ & 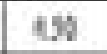 & 1 & $9 x$ & 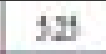 & 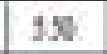 & ij & 48 \\
\hline $1 x$ & EAtit & $m$ & int & 47 & 154 & AIII & $1+4$ & 478 & nuts & +11:? & 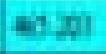 & 744 & 107 & PE III \\
\hline $2 H$ & Hits & 패낸 & ist & 4 & $4 i t$ & 14ty & $13+4$ & 111 & $H$ & thit & 4 & xin & infer & A \\
\hline ift & सiका & 패내 & phe & 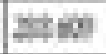 & yis & 164 & 1470 & 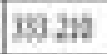 & & int & & & & \\
\hline 14 & thitot & 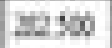 & 24. & WN & 7. & Itity & Dill & motil & Hith & कiti & $4 x$ & that & ytden & ants \\
\hline 14) & Mast & $H$ & Fet & ind & 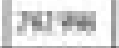 & 404 & 7illat & $0 x$ & 1 & घात & 44: & A $1=7$ & $\pi$ & EH \\
\hline 17. & : & $\tan$ & 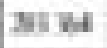 & 44 & जor & WE: & itam & in & WH & hith & WAH & thind & 12: & IHAII \\
\hline $4 \pi$ & Аान & x 64 & & & & 74 & & & & wing & $47+4$ & $y=48$ & & $F+4$ \\
\hline $4 \pi$ & in & 7 & 2 & 1 & 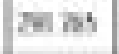 & 1 & & int & & & wit & พอนเ & & \\
\hline 4. & hes. & 1 & t & 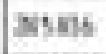 & $\sqrt{14} 4$ & in & itran & Est & & & 1 & 4 & 7 & \\
\hline 47 & ntint & & & & & & & & & & & $4 x+1$ & मи सै: & Hind \\
\hline 10 & $z$ & & & + & $27 x$ & & & & & & & 5 & & \\
\hline $1 \%$ & $=4$ & 14 & 패 & $4=4$ & 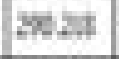 & & 4 & 4 & & 12 & 11 & tit & 证鬲 & ini \\
\hline 10 & 2 & & & & & & & & & $T^{-4}$ & & & te & \\
\hline 13 & 2 & 1 & 7 & $8 x$ & 4 & 14: & 3 & a & 155 & 425 & a & wat & $y$ & 14 \\
\hline 77 & 2 & 4t & al & 474 & $1+$ & $2 x+4$ & $F+1$ & 142 & Hot & $4+14$ & 19 & 747 & ntil. & it: \\
\hline $7 x$ & Wha & 514 & 2713 & ma & as & $y$ & it & & 19 & 4 & & 1 & & in \\
\hline $\mid a$ & MnI & $\cos$ & $2 x$ & 1413 & 134 & 354 & Nat & $3 \pm 4$ & $18 x$ & +1.10 & 4m & wh & 144 & 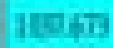 \\
\hline
\end{tabular}

Fig. 3. The balance of $k, T$ and $C t$

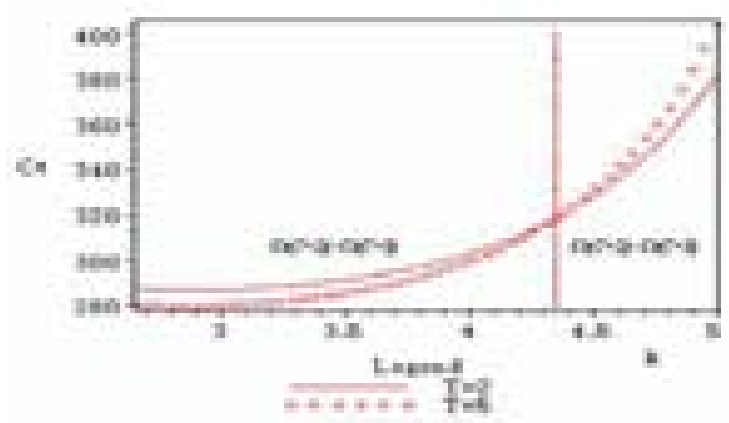

Fig. 4. The relation between $k$ and $C t(T=2, T=$ 5).

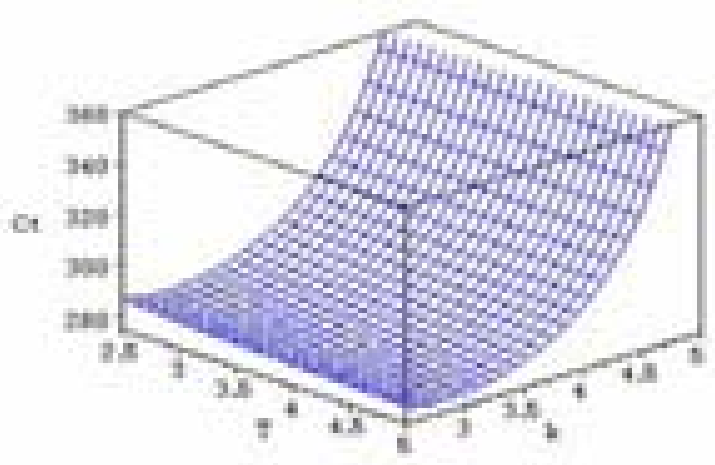

Fig. 5. The relation between $T, k$ and $C t$. 


\begin{tabular}{|c|c|c|c|c|c|c|c|c|c|c|c|c|c|c|}
\hline \multirow{2}{*}{7} & \multicolumn{14}{|c|}{4} \\
\hline & 41 & 011 & $0 x$ & 15 & ass & ba & as & a11 & EB & as & IE & 1tit & bs & 14 \\
\hline 14 & 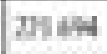 & $70+1$ & тин & $74 \times 12$ & गמल & Mity & 2114 & ruth & M. III & $m+n$ & $74+4$ & th้ & Nitis & MnI \\
\hline $4 \pi$ & 24+15 & HH & Aqu & Has & $2+11$ & $24.4 i$ & FiHt & 7मा1 & $24+3$ & IHE & $20 \mathrm{WI}$ & HA & Dility & 2040 \\
\hline 17 & 21) & 412111 & $24+4$ & 41:4 & $217+1$ & wh: & tyit: & tyuㅏㄹ & 743 & $17+1$ & III & thit: & 73141 & miн \\
\hline 12 & 21:4 & 714 ist & 2014 & \$14t & +4t & Wint & 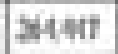 & thati & pyit thi & 17: & Int & मा: & Fath & 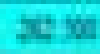 \\
\hline $9 x$ & Dyม & $24+7$ & 2010 & 21\% & ISAl & xals & xust & ans: & 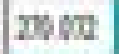 & Intit & Ithe & गhing & mats & NA \\
\hline 97 & 2453 & $24+1$ & 3414 & 24M & $14+4$ & not & Mys & AndT & JWIt & InSt & Then & mith & Itrii & 1440 \\
\hline it & Dys & 14II & $24 \mathrm{nl}$ & 2449 & $\mathrm{NH}$ & $\mathrm{xm}$ & tast & Ant1T & In14 & Inis & 744 & Inte? & Ints & Mt4 \\
\hline 17 & yint & 7414 & 2401 & $24+4$ & 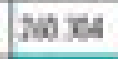 & axist & 7nt4 & trple & $7 x+4$ & that & $74+1$ & सHA1: & 7inth & tats \\
\hline 4: & III. 4 & 4112 & mas & numi & mit 4 & 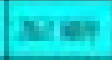 & min & Мнын & $4+4$ & IFH & $74: 4$ & In: & มाก & BuII \\
\hline it & 244 & 27144 & AtMH & 4501 & ans & IFIA & 251 & 77pdx & nind & 2h+4 & Dith & dith & AHIA & Isw \\
\hline $1 \pm$ & IA & भाLT & In 1 & 547E & m.1. & rats: & ItAFE & THII & 개자 & In म & Mrs & In:4 & MHA & HFE: \\
\hline 67 & $1+39$ & I4til & yat & 1417 & 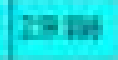 & มี⿻ & tyip & 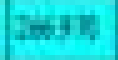 & 배닥 & main & 711. & Ijua & thus & Mald \\
\hline int & Mnei & 94in: & 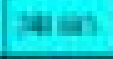 & Fint & interit & ini tia & HII & Homi & ainer & Itil & mis & thint & Mitia & 164 \\
\hline 4 & IIIt) & stin & 7xin & min & with & 파가. & समान & fyiln & min & 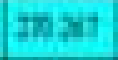 & 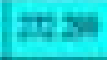 & Ifthy & 페시 & Int \\
\hline in & MHII & if 311 & Iots & EI:W & InI & mEt & HIIA & -344 & mits: & $I+1 \mathrm{HI}$ & HI $\mathrm{Al}$ & WH & Mith & Ir tey \\
\hline 14 & 74 & Arth & 매내 & Hitt & 4nI & $w 5$ & tritil| & thint & xtot & Fi1 & 피내 & ग114 & 하낸 & $14 \mathrm{th}$ \\
\hline 1 & nith & $4 \pi$ & intil & hase. & सम14 & has & vita & 개난 & niil & tक्या & In & $2 p+4$ & $742 \pi$ & Than \\
\hline
\end{tabular}

Fig. 6. The balance of $a, T$ and $C t$

\subsection{The relation between $t, a$ and $c t$}

Fig. 6 shows the relation between the due time and ACT time and the total expectation cost, which is useful for setting the optimal due time and ACT time to the supplier.

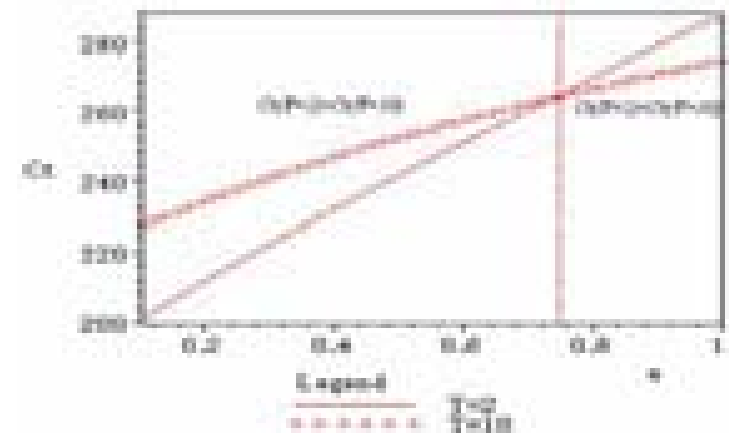

Fig. 7. The relation between $a$ and $C t(T=2, T=$ 10).

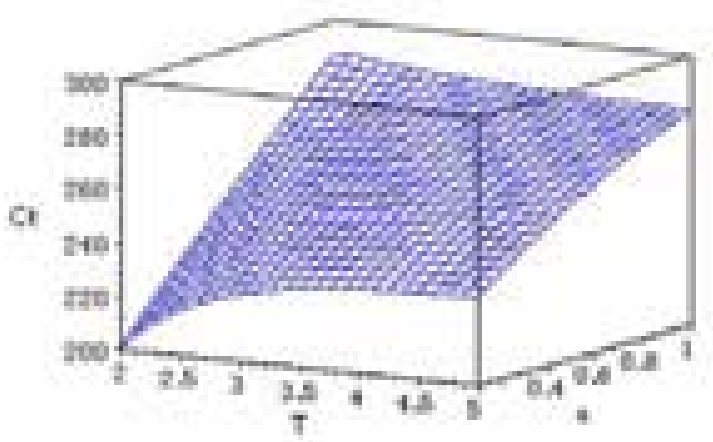

Fig. 8. The relation between $T, a$ and $C t$

From Fig. 6, it can be understood that this tables are divided into two areas by the changed ACT time: in the colorlessness area, the expected total cost per unit time $(C t)$ increases with the increase of due time $(T)$; in the blue area, the expected total cost per unit time $(C t)$ decreases with the increase of due time $(T)$. 
From Fig. 6 and Fig. 7, it can be noted that the expected total cost per unit time $(C t)$ increases with the increase of Act time $(a)$. This is because that the cost of defective goods increases by the increase of ACT time. Also it can be understood that a longer due time should be set when the ACT time is long, while a shorter due time should be set when the ACT time is short from an economic aspect.

In addition, to clarify it more, we also show the Fig. 8 which is the same as the case of Fig. 6.

\section{Conclusions}

In this research, from an economic viewpoint, we present a design of the $\bar{x}$ control chart used in supply chain management system in order to improve customer satisfaction of supplier.

Because of competition in supplier markets, studying the balance of quality and the due time and cost has become a new problem to supplier. To resolve this problem, we showed the mathematical formulations which correspond to this design; moreover, by numerically consideration, we studied the balance of quality (control limits width) and due time and the total expectation cost; and discussed the relations between the due time, ACT time and the total expectation cost.

When due time is short and penalty cost for the delay is larger, some comments are drawn as follows, which would become useful references for setting the optimal due time and control limits width to the supplier.

(1) The expected total cost per unit time $(C t)$ increases with the increase of control limits width $(k)$.

(2) A longer due time should be set when the high quality is demanded from an economic aspect.

(3) The expected total cost per unit time $(C t)$ increases with the increase of Act time $(a)$.

(4) A longer due time should be set when the ACT time is long, from an economic aspect.

\section{References}

[1] D. Bai, M. Lee. An economic design of variable sampling interval $\bar{x}$ control charts. International Journal of Production Economics, 1998, 54: 57-64.

[2] E. Castillo, D. Montgomery. A general model for the optimal economic design of $\bar{x}$ charts used to control short or long run processes. IIE Transactions, 1996, 28: 193-201.

[3] S. Crowder. An spc models for short production run: minimizing expected cost. Technometrics, 1992, 34: 64-73.

[4] A. Duncan. The economic design of charts used to maintain current control of a process. Journal of the American Statistical Association, 1956, 51: 228-242.

[5] I. Gibra. Economically optimal determination of the parameters of control charts. Management Science, 1971, 17: 635-647.

[6] T. Ikezawa. I have leaned from tqc. Quality Management (In Japanese), 1985, 36(1): 6-12.

[7] L. Jones, K. Case. Economic design of a joint $\bar{x}$ and $\mathrm{r}$ chart. IIE Transactions, 1981, 13: 182-195.

[8] N. Kano. Historical progress of the birth of pdca cycle, problem solving qc story and task achieving qc story. in: Proceedings on the Research Conference of Japanese Society for Quality Control (in Japanese), 2006, 7-10.

[9] S. Ladany, D. Bedi. Selection of the optimal setup policy. Naval research Logistics Quarterly, 1976, 23: 219-233.

[10] M. Matsui. A management cycle modle: "switching control under lot processing and time span". Journal of Japan Industrial management Association, 2005, 56: 256-264.

[11] E. Sanige. The economic statistical design of control charts with an application to $\bar{x}$ and $\mathrm{r}$ chart. Technometrics, 1986, 28: 3-10.

[12] W. Shewhart. Economic Control of Quality of Manufacturing Product. Van Nostrand, New Jersey, 1931.

[13] S. Shiba, D. Walden. Four practical revolutions in management: Systems for creating unique organizational capability. in: Productivity Pr., 2001.

[14] J. Sun, M. Tsubaki, M. Matsui. The mathematical formulations of pdca quality control model of $\bar{x}$ control charts. in: Proceedings on the Research Conference of Japan Industrial Management Association (in Japanese), 2003, $16-17$.

[15] J. Sun, M. Tsubaki, M. Matsui. Economic models of $\bar{x}$ chart with tardiness penalty in finite due time processes. Journal of Japan Industrial management Association, (in Japanese), 2006, 57(5): 374-387. 